\title{
The impact of ELMs on the ITER divertor
}

\author{
A.W. Leonard ${ }^{\mathrm{a}, *}$, A. Herrmann ${ }^{\text {b }}$, K. Itami ${ }^{\text {c }}$, J. Lingertat ${ }^{\mathrm{d}}$, A. Loarte ${ }^{\mathrm{e}}$, \\ T.H. Osborne ${ }^{a}$, W. Suttrop ${ }^{\mathrm{e}}$, the ITER Divertor Modeling and Database Expert \\ Group ${ }^{f}$, the ITER Divertor Physics Expert Group ${ }^{f}$ \\ ${ }^{a}$ General Atomics, P.O. Box 85608, San Diego, CA 92186-5608, USA \\ ${ }^{\mathrm{b}}$ Max Planck Institute of Plasmaphysics, Garching, Germany \\ ${ }^{\mathrm{c}}$ Japan Atomic Energy Research Institute, Naka, Japan \\ d Jet Joint Undertaking, Abingdon, UK \\ e Next European Torus (NET), Garching, Germany \\ ${ }^{\mathrm{f}}$ ITER Organization, San Diego, CA, USA
}

\begin{abstract}
Edge-Localized-Modes (ELMs) are expected to present a significant transient flux of energy and particles to the ITER divertor. The threshold for ablation of the graphite target will be reached if the ELM transient exceeds $Q /$ $t^{1 / 2} \sim 45 \mathrm{MJ} \mathrm{m}^{-2} \mathrm{~s}^{-1 / 2}$ where $Q$ is the ELM deposition energy density and $t$ the ELM deposition time. The ablation parameter in ITER can be determined by scaling four factors from present experiments: the ELM energy loss from the core plasma, the fraction of ELM energy deposited on the divertor target, the area of the ELM profile onto the target, and finally the time for the ELM deposition. Review of the ELM energy loss of Type I ELM data suggests an ITER ELM energy loss of 2-6\% of the stored energy or 25-80 MJ. The fraction of heating power crossing the separatrix due to ELMs is nearly constant $(20-40 \%)$ resulting in an inverse relationship between ELM amplitude and frequency. Measurements on DIII-D and ASDEX-Upgrade indicate that $50-80 \%$ of the ELM energy is deposited on the target. There is currently no evidence for a large fraction of the ELM energy being dissipated through radiation. Profiles of the ELM heat flux are typically 1-2 times the width of steady heat flux between ELMs, with the ELM amplitude usually larger on the inboard target. The ELM deposition time varies from about $0.1 \mathrm{~ms}$ in JET to as high as $1.0 \mathrm{~ms}$ in ASDEXUpgrade and DIII-D. The ELM deposition time for ITER will depend upon the level of conductive versus convective transport determined by the ratio of energy to particles released by the ELM. Preliminary analysis suggests that large Type I ELMs for low recycling H-mode may exceed the ablation parameter by a factor of 5. Promising regimes with smaller ELMS have been found at other edge operational regimes, including high density with gas puffing, use of RF heating and operation with Type III ELMs. (c) 1999 Elsevier Science B.V. All rights reserved.
\end{abstract}

Keywords: ELM; ITER; Divertor erosion

\section{Introduction}

Edge-Localized-Modes (ELMs) are a common feature of H-mode operation in most tokamaks [1-4]. The ELM instability relieves the plasma pressure gradient that builds just inside the separatrix and releases energy and particles into the Scrape-Off-Layer (SOL) in a very short timescale, $<1 \mathrm{~ms}[5,6]$. Although ELMs help re-

\footnotetext{
* Corresponding author. Tel.: +1 619455 2214; fax: +1 619 455 4156; e-mail: leonard@gav.gat.com
}

lieve buildup of particles and impurities in the main plasma, the effect of ELMs on the divertor has not been widely investigated. ELMs are of concern to ITER because of the transient heat and particle flux that can be deposited on the divertor target. Heat flux transients are of particular concern because significant target plate erosion due to vaporization can occur if the surface temperature rises high enough. Since a large number of ELMs, $\geqslant 1000$, are expected in each discharge it is important that the surface temperature rise due to an individual ELM remain below this threshold. 
If the carbon divertor target surface temperature rises above about $2500^{\circ} \mathrm{C}$ then excessive erosion due to vaporization can take place. In a 1-D approximation an energy density, $\Delta Q$, deposited in time $t$ will cause the surface temperature to rise as $\Delta T \propto Q t^{-1 / 2}$. More accurate calculations have been carried out for the ITER divertor [7] to predict the lifetime of the ITER divertor when subject to heat flux transients, such as ELMs or disruptions. If the ELM energy density on the target exceeds a threshold for ablation the target lifetime may be no more than about $10^{4}$ ELM events. This threshold is $\sim 0.5 \mathrm{MJ} / \mathrm{m}^{2}$ if the ELM energy is deposited in $0.1 \mathrm{~ms}$ or $1.2 \mathrm{MJ} / \mathrm{m}^{2}$ if the deposition time is $1.0 \mathrm{~ms}$. Using the 1-D $t^{-1 / 2}$ dependence an ablation threshold can be estimated at $Q t^{-1 / 2} \approx 45 \mathrm{MJ} \mathrm{m}^{-2} \mathrm{~s}^{-1 / 2}$ where $Q$ is the energy density deposited on the target for an individual ELM in $\mathrm{J} \mathrm{m}^{-2}$ and $t$ is the time in seconds for that deposition. If a significant fraction of ELMs exceed this threshold then an unacceptable level of erosion can take place.

The location of the ELM flux is of concern as well. If the ELM energy falls on a location far from the nominal strike point then it may land on a surface not designed to handle high power and may cause unacceptable damage. The ELM particle flux profile is important also. The ITER divertor is designed to baffle neutral particles to keep them from recycling into the main chamber. If the ELM particle flux has a different spatial distribution than the steady-state profile between ELMs then more neutrals might escape to the main chamber. This could require a change in design of the divertor in order to better baffle the neutrals from the ELM particle flux.

In order to assess the potential impact of ELMs on the ITER divertor a database of ELM characteristics was compiled from the world's tokamaks. This effort was carried out under the organization of the ITER Divertor Modeling and Database Expert Group. Data on ELMs was collected from ASDEX-Upgrade, DIII-D, JET, JT-60U and COMPASS and assembled into a database. This data as well as previously published results are studied to predict ELM behavior in ITER.

The greatest concern is that ELMs will lead to unacceptable erosion of the ITER divertor. Such erosion will occur if the ELM heat flux parameter exceeds the threshold of $45 \mathrm{MJ} \mathrm{m}^{-2} \mathrm{~s}^{-1 / 2}$. One can hope to predict the ITER ELM heat flux parameter if the scaling of four parameters can be determined: (1) (Section 2) the energy lost from the main plasma due to an individual ELM, (2) (Section 4) the fraction of this energy that is deposited on the target, (3) (Section 3) the area over which the ELM energy is deposited, (4) (Section 5) and finally the time duration of the ELM heat flux. In this paper we will examine the existing data to determine how well we can predict for ITER these four parameters. We find that for ELMing H-mode at the natural density the ELM heat flux is likely to be above the threshold for ablation, but several operating scenarios may produce lower, acceptable ELM heat flux. We will then comment on the implications for ITER operation.

\section{ELM energy loss}

During H-mode the pressure gradient just inside the separatrix rises to a high level. An instability, commonly believed related to the ideal ballooning limit $[5,8]$ relieves the pressure buildup by releasing energy and particles into the SOL on a very fast timescale, $<1 \mathrm{~ms}$ [9-11]. It is the energy lost from inside the separatrix that flows along field lines into the divertor resulting in the ELM heat flux pulse. The first objective is to determine the magnitude of energy loss from the main plasma due to individual ELMs.

The magnitude of energy lost from the core plasma, or ELM energy, has been measured on several tokamaks to be in the range of $2-6 \%$ of the main plasma stored energy $[15,18]$. These measurements from JET, ASDEXUpgrade, DIII-D and COMPASS-D are for TYPE I ELMs in H-mode with no additional gas puffing. As the heating power was increased the ELM energy remained constant while the ELM frequency increased linearly with heating power. This resulted in a nearly constant fraction of the heating power, $20-40 \%$, being carried across the separatrix by ELMs [15-19]. For an ITER stored energy of $1200 \mathrm{MJ}$ and a power of $200 \mathrm{MW}$ crossing the separatrix this data would indicate an ITER ELM energy between 25 and $80 \mathrm{MJ}$ with an ELM frequency of approximately $1-5 \mathrm{~Hz}$.

The ITER ELM database has been assembled to aid in prediction of the ELM characteristics for ITER. The database has sought contributions from the world's tokamaks for ELM characteristics such as ELM energy and frequency as well as other global steady-state parameters. At this time the database contains ELM energy data only from JET and DIII-D, while ELM frequency data has been obtained from JET, DIII-D, JT-60U and ASDEX-Upgrade. Measuring the energy loss from an individual ELM can be difficult because the $2-6 \%$ of the main plasma energy that is lost can easily be within the scatter of the measurement. JET determines the ELM energy with diamagnetic loop measurements while data from DIII-D is based on equilibrium reconstruction from magnetic probes. The contributed ELM energy data covers a range of main plasma parameters for H-mode with Type I ELMs. For JET, data has been contributed for $I_{\mathrm{p}}=1.6-4.8 \quad \mathrm{MA}, \quad B_{\mathrm{t}}=1.5-3.5 \quad \mathrm{~T}$, $q_{95}=2.4-3.6$ and $P_{\text {in }}=6.5-21.9 \mathrm{MW}$. The DIII-D data includes $I_{\mathrm{p}}=0.8-1.8 \mathrm{MA}, B_{\mathrm{t}}=1.1-2.1 \mathrm{~T}, q_{95}=3.2-8.5$ and $P_{\text {in }}=2.4-15.2 \mathrm{MW}$. The variation in plasma shape parameters such as triangularity is smaller, though shape should play an important role because of its influence on edge stability. The preliminary state of the 
database makes it difficult to predict ELM characteristics for ITER with any level of confidence. Until further refinement and analysis of the database is obtained, the earlier estimate for Type I ELMs in ITER remains the best available.

With only JET and DIII-D reporting ELM energy and initial analysis of the data only begun, the database is most useful for examining common relationships existing in different tokamaks. Since ELMs are related to stability of the steep gradient region just inside the separatrix, initial analysis of the database has focused on examining relationships between the edge characteristics and the ELM energy. Previous work on DIII-D [12] has shown the energy of individual ELMs to be nearly proportional to the edge pressure pedestal. It was found on DIII-D that the ELM energy was approximately 1/3 of the pedestal electron energy. The pedestal electron energy is given by the value of the electron pressure, at the top of the steep gradient region just inside the separatrix, multiplied by the entire plasma volume inside the separatrix. To determine if the ELM energy is more universally a constant fraction of the edge pedestal energy, the edge pedestal characteristics for JET and DIII$\mathrm{D}$ are compared to the ELM energy. In Fig. 1 we plot the ELM energy versus the pedestal electron energy as defined above. We find that the ELM energy is $\sim 36 \%$ of
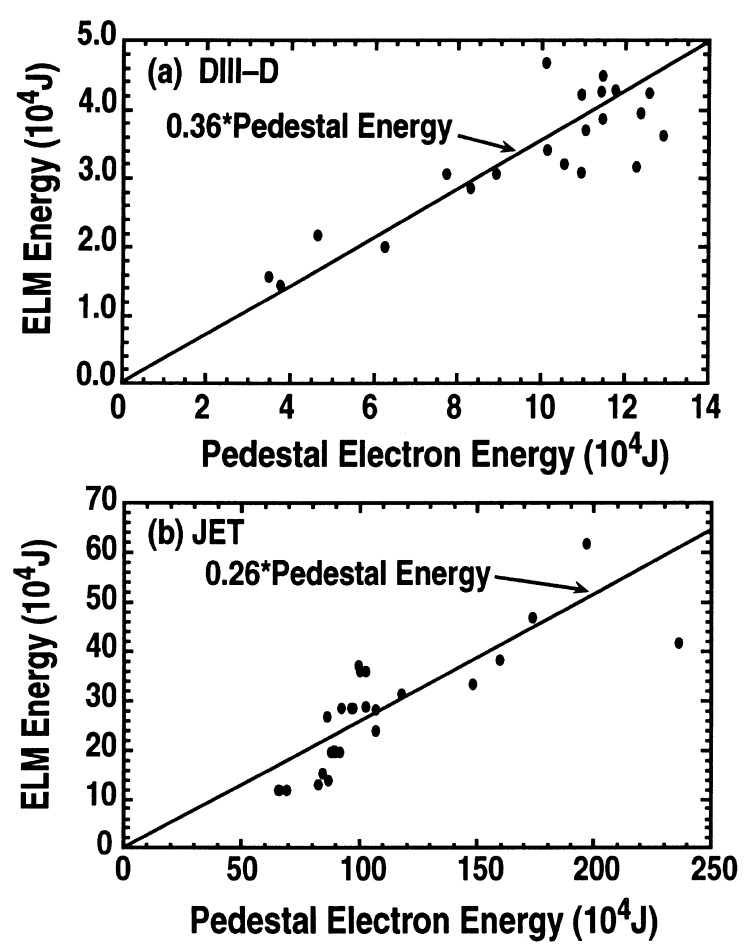

Fig. 1. The ELM energy loss, $\Delta W$, versus the pedestal electron pressure integrated over the plasma volume for: (a) DIII-D; (b) JET. the pedestal electron energy in DIII-D and $\sim 26 \%$ in JET. It is interesting that this fraction remains nearly constant over a wide range of main plasma parameters. The data is too sparse and with too much scatter, however, to determine if the ELM fractional pedestal energy loss is independent of machine size or scales like $R^{-1 / 2}$ as implied by the linear fit. Also, the size of the ELM energy is a surprisingly large fraction of the pedestal energy. The amplitude of the ELM implies that the ELM instability transports energy from far inside of the steep gradient region where the ELM instability is thought to originate.

Maintaining a high temperature at the top of the edge pedestal is thought to be important in achieving optimal confinement in the main plasma [13]. A pedestal temperature of $3-4 \mathrm{keV}$ has been projected to provide adequate ITER performance. Given ITER's proposed operating density and plasma volume this would represent an ELM energy of approximately $25 \mathrm{MJ}$ if the same fraction of pedestal energy was lost in ITER as was found in DIII-D and JET. Clearly more work is needed to understand what factors may control the relationship between ELM energy and the edge pedestal characteristics.

There are examples of smaller ELM amplitude that are clearly less than the $2-6 \%$ of the plasma stored energy previously reported. This has most often been demonstrated by additional gas puffing into an ELMing $\mathrm{H}$-mode plasma. DIII-D has reported a factor of 4 or more reduction in ELM amplitude and similar increase in ELM frequency with external gas puffing [12]. As of yet there is no scaling of ELM amplitude or frequency with main plasma fueling or plasma density. The reduction in ELM amplitude with gas puffing is usually accompanied by a reduction in the edge pressure pedestal [14]. Reduced pedestal parameters have been associated with a reduced level of confinement in the main plasma. A penalty in confinement may then be expected if such a means is used to reduce the ELM amplitude. A mode of $\mathrm{H}$-mode operation with RF heating was found on JET whereby the ELM amplitude was reduced a factor of 5 or more yet did not degrade confinement of the main plasma [20].

Other regimes of edge operation may lead to an entirely different class of ELM. When operated near the Hmode threshold Type III ELMs have been observed [21]. The energy loss of the Type III ELMs have not yet been measured as they are much smaller than the Type I ELMs. Signal levels from diamagnetic diagnostics and divertor heat flux would indicate the Type III ELMs are more than an order of magnitude smaller than the Type I ELMs.

\section{ELM heat flux profile}

Energy released by the ELM instability into the SOL is transported along field lines before arriving at the 
divertor target. The energy density on the target due to the ELM will depend on the area, or profile, over which the energy is deposited. This profile can be very different from the steady-state heat flux between ELMs. Divertor target heat flux is typically determined by IR camera measurements [22] of the divertor surface temperature. The time dependent surface temperature can then be unfolded to determine heat flux to the surface. This can be problematic for the short but intense ELM heat pulse. The existence of a thin surface layer of amorphous graphite with a reduced thermal conductivity could result in overestimating the instantaneous heat flux of such a pulse. ASDEX-Upgrade has invoked such a surface layer to explain the time behavior of their divertor surface temperature measurements [23]. The total energy deposited, and the profile of that energy is much less susceptible to such errors. Measurements of the ELM energy deposition profile have been made on ASDEXUpgrade, DIII-D, JET and JT-60U. Examples of an ELM profile from each of the tokamaks are shown in Fig. 2. The profile for JET in Fig. 2(c) shows the divertor surface temperature during an ELM and between ELMs.
A common feature of the ELM profiles is the effective width of the ELM heat flux is of the same order to twice the width of the steady-state heat flux between ELMs. The data from DIII-D and ASDEX-Upgrade plot the total heat flux profile averaged over ELMs. In the outer divertor the total heat flux profile is indicative of that between ELM profile because the time-averaged ELM flux represents a small part of the total. Although the steady-state data between ELM heat flux from JT-60U is not shown in Fig. 2, other measurements on these tokamaks as well as COMPASS-D [18] confirm these width observations.

Comparisons of the heat flux profile during and between ELMs has been made in only a couple of cases. It has not yet been determined if the ELM profile varies similar to the between ELM, or steady-state, profile with variations in parameters such as plasma current, density and toroidal field. The variation of the steady-state heat flux width with these parameters is a subject still under study.

The width of the ELM heat flux is determined by the competition between parallel and perpendicular transport, just as the steady-state width is determined. The
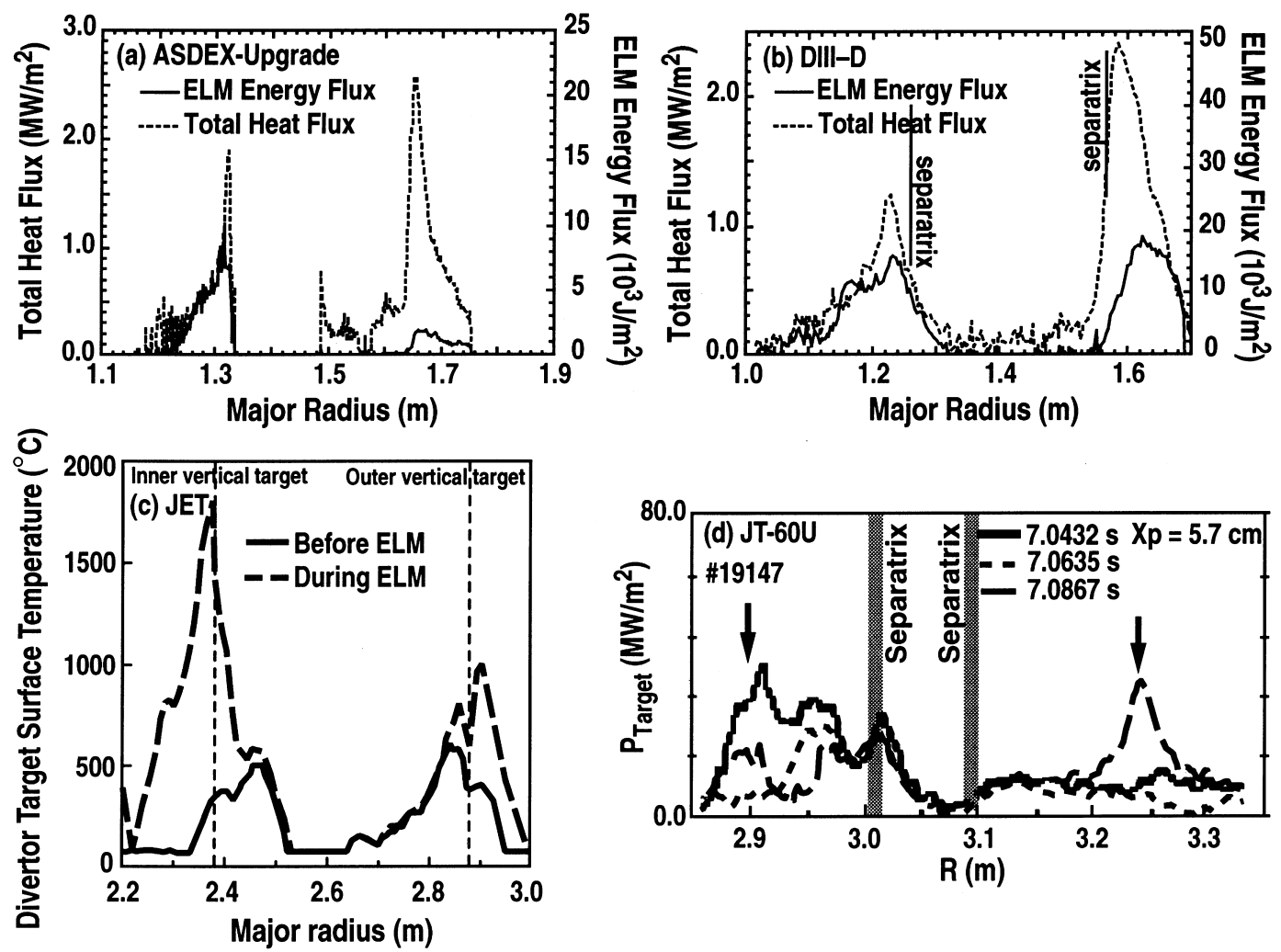

Fig. 2. ELM deposition profile for: (a) ASDEX-Upgrade; (b) DIII-D; (c) JET; (d) JT-60U. For ASDEX-Upgrade and DIII-D the total heat flux profile averaged over ELMs is also shown. The JET profile shows the surface temperature between and during ELMs. The three ELM profiles shown for JT-60U illustrate the variability of the profile for individual ELMs. 
fast parallel transport should be governed by the same processes as during the steady-state phase. One would then expect the ELM heat flux width to be somewhat wider than the steady-state width depending on the level of additional transport the ELM instability introduces. There is an increased level of magnetic fluctuations during the ELM, but no study has been reported as to the correlation of fluctuation level to ELM energy flux profile. Some of the irregular features of the ELM profiles shown in Fig. 2 could be due to the turbulent nature of the ELM instability. The irregular nature of the ELM profile makes a definition of width difficult for crossmachine comparison. With no size scaling in the width ratio apparent in the present data, our best estimates are that the ITER ELM width will be a factor of 1-2 greater than the heat flux width between ELMs. A more detailed study of the widths with correlation to other plasma parameters and the ELM instability itself for projections to ITER remains a subject of future work.

As clearly seen in the ELM profiles, the ELM heat flux is typically reported as being a factor 2-4 larger on the inboard divertor target than the outboard. Such an in/out asymmetry would result in an effective decrease, by up to a factor of 2, the area over which the ELM energy is deposited. Time-dependent 2D simulations of ELMs [24] have been able to produce moderately more influx to the inboard divertor target. The larger inboard heat flux is attributed to expansion of the inboard flux surfaces just inside the separatrix. These simulations also assumed the ELM instability resulted from poloidally symmetric enhanced transport.

An alternative explanation for part of the in/out asymmetry comes from the interpretation of the divertor surface temperature measurements. As stated earlier, a surface layer of lower conductivity will result in a greater temperature rise of the surface for a given energy deposited. Analysis of the divertor tiles of ASDEX-Upgrade [25], JET [26] and DIII-D [27] all reported that after a period of operation a much greater retention of deuterium was found at the inboard divertor as compared to the outboard. This could be attributed a layer of redeposited amorphous graphite on the target plates with a much thicker layer on the inboard compared to the outboard. This amorphous layer presumably has a much lower conductivity than the graphite base material and would lead to a greater rise in surface temperature for the inboard target compared to the outboard for the same ELM energy deposition. Whereas different thickness layers would likely not affect estimates of heat flux which have been averaged over times much longer than the ELM duration, the estimate of energy deposited during a single ELM might be affected. More analysis is needed to determine to what extent the observed in/out asymmetries could be explained by differences in the divertor plate surface properties. Other measurements [28,36], from divertor target Langmuir probes and spectroscopic profile measurements, indicate that some degree of ELM in/out asymmetry does exist.

Toroidal asymmetries could also reduce the effective area over which the ELM energy is deposited. Simultaneous measurements of ELM energy deposition were made at two different toroidal locations on DIII-D [15]. These measurements showed toroidal peaking of less than $50 \%$, which was within the measurement uncertainty. This magnitude of toroidal asymmetry would result in a reduction of the effective ELM area by no more than $1 / 4$.

Using the profile factors discussed above, and assuming only a modest in/out asymmetry, the ELM deposition area on ITER should range from 1 to 2 times that of the steady-state heat flux between ELMs. For ITER the steady-state heat flux area is expected to be about $10 \mathrm{~m}^{2}$, leading to an ELM area of about 10-20 $\mathrm{m}^{2}$. More work will be needed to reduce this uncertainty and to refine the estimate of the peak energy density as defined by ELM energy loss divided by the ELM deposition area.

Another concern for the ELM energy flux deposition is the location of the deposition with respect to the separatrix. If the ELM energy falls far from the separatrix then it may land on invessel components that are not designed for high heat flux. A shift in the ELM profile can be seen in the JET data of Fig. 2(c). JET has reported that the ELM energy to the inboard divertor may fall as much as $20 \mathrm{~cm}$, a distance of several SOL widths, from the steady-state location of the inboard separatrix [28]. Such a large shift in ITER could damage surfaces outside the divertor structure. The shift in JET appears to be toroidally symmetric. Current redistribution at the ELM might account for such a rapid shift in the plasma magnetic structure. Currents of sufficient magnitude to produce such a shift have been measured at the divertor target. This phenomena is not well understood, but does appear to decrease in magnitude with smaller amplitude ELMs on JET [29].

\section{ELM dissipation}

If a significant fraction of the ELM energy can be radiated then the possibility exists for reducing the energy flux onto the target. The radiation must be intense enough so that a significant fraction of the ELM energy is lost within the duration of the short ELM heat pulse onto the target plate. Measurements of ELM radiation on DIII-D [15] have shown that less than $20 \%$ of the ELM energy is lost as radiation. Radiation measurements on JET [30] have shown that the ELMs cause additional radiation, but that radiation rises after the fast heat pulse. ELM divertor heat flux measurements on ASDEX-Upgrade [31] and DIII-D [15] have measured between $50 \%$ and $80 \%$ of the ELM energy loss 
deposited on the divertor target. However there is large uncertainty in these measurements due to difficulty with divertor surface temperature interpretation, as stated earlier, and measurement uncertainties of the ELM energy loss itself.

A possibility for ELM radiation is for the ELM heat pulse to cause high levels of noncoronal radiation in impurities in the divertor. The fast rise in temperature in the divertor will ionize the low charge state impurity ions and could result in significantly enhanced radiation. To estimate what would be required, assume $20 \mathrm{MJ}$ of the ELM energy is to be dissipated in $1 \mathrm{~ms}$ giving a radiative power level of $20 \mathrm{GW}$. If carbon is the dominant impurity in the divertor it is difficult to envision achieving this power level even with high impurity fractions and noncoronal radiation enhancement. However, to properly assess this process, time-dependent modeling with the proper atomic physics is necessary. Simulations of ELMs have been carried out using the B2/EIRENE code $[32,33]$. This study found that much less than $1 \mathrm{MJ}$ could be radiated for ITER conditions. This level of radiation would be a significant fraction of the ELM energy only if the ELMs are small enough to be tolerable even without additional radiation.

It is possible that some fraction of the ELM energy may be lost through other means. Because of the highly turbulent nature of the ELM instability it is possible that some fraction of the ELM energy is transported to flux surfaces far from the separatrix. Fast visible camera images on JET [34] during ELMs have shown clearly visible interaction with the inside wall and other surfaces. It is likely this interaction represents only a small fraction of the ELM energy.

With divertor measurements on DIII-D and ASDEX-Upgrade accounting for $50-80 \%$ of the ELM energy and prospects for ELM radiation difficult, it seems prudent to assume that most of the ELM energy on ITER will be deposited on the divertor target.

\section{ELM duration}

The short duration of the ELM heat pulse increases the potential for target plate ablation. If the heat pulse for a given energy deposition occurs in a shorter time, the divertor surface temperature will increase as $t^{-1 / 2}$. If the ELM instability in the main plasma is of short duration, then the duration of the divertor target heat pulse will be determined by parallel transport processes. If only energy is released by the ELM instability there will be no change in density profiles, but the plasma inside the separatrix will be cooled while the plasma in the SOL will be heated. This energy can be transported to the divertor very fast, on the order of the electron thermal speed. If part of the ELM energy is in the form of extra density released into the SOL then the timescale for transport of this energy is the time for redistribution of particles, or approximately the ion thermal speed.

High speed measurements of the JET divertor target show that most of the ELM energy can be deposited on the target in as little as about $100 \mu \mathrm{s}$ [35]. An example of the time dependence of the surface temperature during an ELM on JET is shown in Fig. 3(a). The temperature rises very quickly, coincident with the ELM instability, and peaks within about $100 \mu \mathrm{s}$. (Time $t=0$ is the beginning of the data record.) An attempt was made to model this time behavior using the 1-D fluid code SOLOne [36]. In this simulation a given amount of energy was fed into the SOL in a short time keeping the number of particles fixed. The time behavior of the divertor conditions, including the target plate heat flux, were then followed. It was found that most of the additional energy was conducted to the target and deposited in about $100 \mu$ s. The fixed particle constraint appears a good match to JET conditions. JET has reported that ELMs with no additional gas puffing do not significantly affect the density profile by releasing particles into the SOL, but do transport heat by reducing the temperature inside the separatrix [37].

The time for ELM heat flux deposition on ASDEXUpgrade and DIII-D has been reported to be longer, up
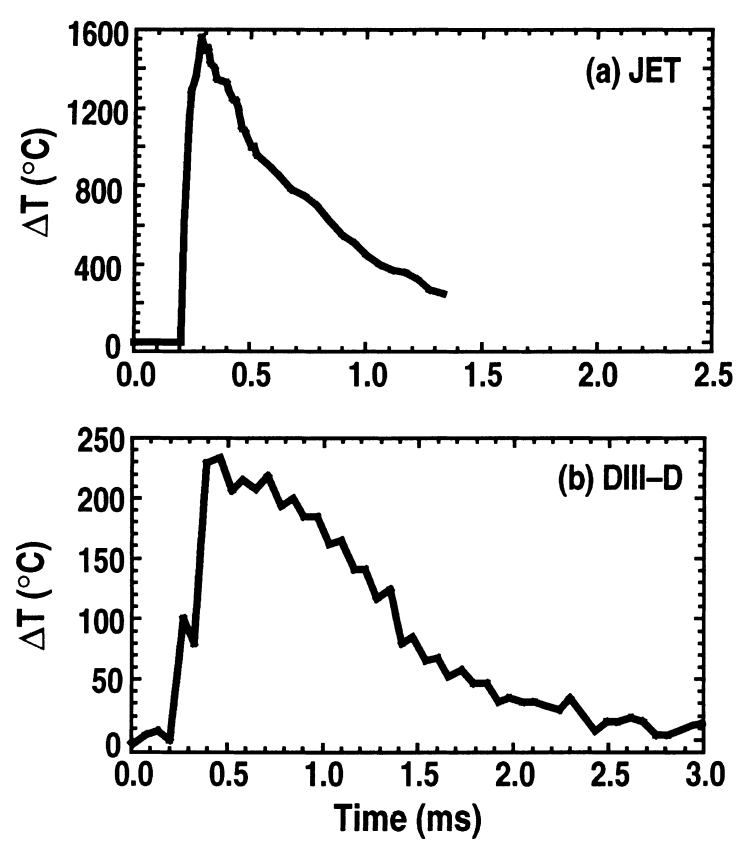

Fig. 3. The temperature rise of the divertor surface as a function of time for: (a) JET; (b) DIII-D. Time $t=0$ is the begining of the data record. The temperature rise is coincident with onset of the ELM instability, within measurement uncertainty. Most of the temperature rise due to an ELM occurs in about $100 \mu \mathrm{s}$ for JET and within $300 \mu$ s for DIII-D. 
to $1.0 \mathrm{~ms}$ [15]. An example from DIII-D is shown in Fig. 3(b). These tokamaks have also reported that ELMs release a significant fraction of their energy as particles into the SOL [12]. The energy associated with additional particles in the SOL will result in a longer thermal equilibration time and is likely the reason for the longer ELM durations on DIII-D and ASDEXUpgrade.

Careful measurement of the ELM heat flux duration has been made in only a few instances. Variations in the duration on a single tokamak have not been measured for example with changes in main plasma density which could affect the fraction of energy that is conducted or convected. These studies must be done in order to verify the conjectures made above.

Predicting the ELM heat flux duration for ITER is then dependent on the ELM instability itself. If an ELM in ITER does not redistribute particles but only releases energy into the SOL, then the ELM heat flux duration can be expected to be the order $100 \mu$ s. If, however, an ITER ELM releases a significant fraction of its energy as additional particles into the SOL, then a longer timescale of 1-2 ms would be expected. Simulations with 2D time-dependent codes should be able to predict the time behavior if these boundary conditions are known. Again, further understanding of ITER edge plasma operation is required to reduce the uncertainty. However, our current uncertainty of the ELM heat flux duration results in only a factor of 3-4 uncertainty in the ablation parameter because of the $t^{-1 / 2}$ dependence. This is the same order as our uncertainty in profile effects on reaching the ablation threshold.

\section{Implications for ITER}

Using the ELM characteristics described above we now project the range of ELM amplitudes that is likely to cause divertor target ablation on ITER. For the area of the ELM deposition we will assume approximately 12 times the steady-state heat flux area between ELMs. Peaking of the ELM energy flux due to effects such as inboard/outboard or toroidal asymmetries appear to be less than a factor of 2, while radiation or other processes should dissipate less than half the ELM energy. We then expect an effective ELM area of 1-2 times the expected steady-state target plate heat flux width on ITER, or 10 $20 \mathrm{~m}^{2}$. An ELM duration time of $0.1-1.0 \mathrm{~ms}$ coupled with the ablation threshold of $45 \mathrm{MJ} \mathrm{m}^{-2} \mathrm{~s}^{-1 / 2}$ leads to an ablation threshold ELM energy range of 5-30 MJ. This is the range of ELM amplitude where we expect excessive divertor plate ablation to begin.

Although the projected ELM energy of 25-80 MJ is much less than the allowable 5-30 MJ, there are reasonable prospects for achieving smaller ELM amplitude in ITER. The range of ELM amplitude of 25-80 MJ was based on H-mode with out extra gas puffing, at low to average density. However, the proposed operation of ITER is at high density near the Greenwald limit. Operation at high density with additional gas puffing often reduces the ELM amplitude a factor of 5 or more. Replacing neutral beam heating with RF on JET showed a decrease in ELM amplitude by as much as a factor of 10 . Use of pellets also has the potential to produce more favorable ELMs [38]. Or, if operation is achieved with Type III ELMs then the ELM amplitude could be more than an order of magnitude reduced from current projections.

The allowable ELM amplitude also has implications for the main plasma performance. Recent work has highlighted the relationship between the edge pressure pedestal and confinement in the main plasma [13]. A high pedestal temperature is thought important for achieving optimal confinement. However, our earlier scaling showed the ELM amplitude increased as the pedestal pressure increased. To estimate an acceptable pedestal temperature we take $30 \%$ of the pedestal electron energy as the value of the ELM energy. An acceptable ELM of $10 \mathrm{MJ}$ gives a pedestal electron energy of about $30 \mathrm{MJ}$. Using the proposed ITER operating density of $\sim 1.0 \times 10^{20} \mathrm{~m}^{-3}$ and a volume of $2000 \mathrm{~m}^{3}$

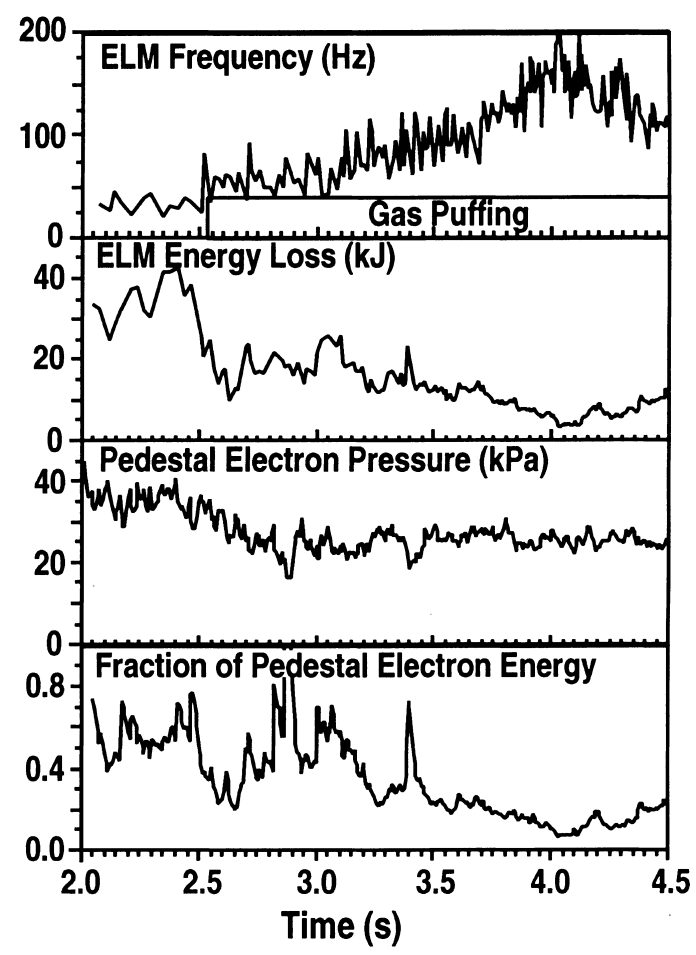

Fig. 4. The ELM and edge pedestal characteristics on DIII-D with gas puffing. Shown are the ELM frequency, the energy loss of individual ELMs, the pedestal electron pressure and the fraction of pedestal energy lost at each ELM. 
leads to a pedestal electron temperature of a little less than $1 \mathrm{keV}$. This is significantly below the $3-4 \mathrm{keV}$ pedestal temperature thought necessary to achieve ITER's optimal performance [13]. If the fraction of pedestal electron energy lost with each ELM actually scales as $R^{-1 / 2}$, as the data indicates it might, then a pedestal temperature of $2 \mathrm{keV}$ might be acceptable. The value is still somewhat low. What is needed is operation with high pedestal parameters that do not drop so significantly during an ELM. An example of the direction of what is needed is shown in Fig. 4. This data from DIII-D [12] shows what can happen to the ELM, pedestal and confinement characteristics. For this case additional gas puffing into an $\mathrm{H}$-mode with Type I ELMs led to an increase in ELM frequency and reduction in ELM amplitude by a factor of 5. The average height of the pedestal though does not decrease nearly so much as the ELM energy. This leads to only a modest degradation in confinement. It is unknown why in this case the ratio of ELM energy to pedestal energy is much reduced. For other cases of gas puffing into H-mode this ratio remains constant. This topic will be the subject of future work.

\section{Conclusions}

Here we have presented a frame work for evaluating the potential ablation of the ITER target due to ELMs. Our initial projections of the ELM amplitude, profile and duration of the heat pulse, indicate that for plasmas at low to moderate density without additional gas puffing and with Type I ELMs the ELM heat flux is likely to be above the target ablation threshold. Though this is just an initial attempt at multi-machine analysis it clearly indicates that standard $\mathrm{H}$-mode operation at low to moderate density is likely to be unacceptable. However, ITER's planned operation is at high density where ELMs have exhibited very different behavior, from large compound ELMs, to rapid smaller amplitude Type I ELMs, to very small amplitude Type III ELMs. Future work on confinement, as well as edge and divertor issues, should concentrate on studying regimes of smaller ELM amplitude that may have acceptable pedestal and confinement characteristics. The greatest uncertainty exists in the characteristics of the ELM instability itself. The modeling tools appear adequate to simulate ELM effects in the SOL and divertor once the ELM instability has been characterized.

\section{Acknowledgements}

The authors wish to acknowledge fruitful discussion with members of ITER Divertor Modeling and Database Expert Group and ITER Divertor Physics Expert
Group. The databases used in the present analysis were supplied by tokamak teams from ASDEX-Upgrade, COMPASS-D, DIII-D, JETand JT-60U. The authors wish to express their sincere thanks to all members of these teams. Computational support by the IPP Information Department and the computer supporting staff of ITER Garching JWS is also acknowledged. Support by US Department of Energy under Contract No. DEAC03-89ER51114, is acknowledged.

\section{References}

[1] F. Wagner, G. Becker, K. Behringer et al., Phys. Rev. Lett. 49 (1982) 1408.

[2] S.M. Kaye, M.G. Bell, K. Bol et al., J. Nucl. Mater. 121 (1984) 115.

[3] N. Ohyabu, K.H. Burell, J.C. DeBoo et al., Nucl. Fusion 25 (1985) 49.

[4] E.J. Doyle, R.J. Groebner, K.H. Burell et al., Phys. Fluids B 3 (1991) 2300.

[5] P. Gohil, M.A. Mahdavi, L.L. Lao et al., Phys. Rev. Lett. 61 (1989) 1603.

[6] H. Zohm, T.H. Osborne, K.H. Burrell et al., Nucl. Fusion 35 (1995) 543.

[7] H.D. Pacher, E9. Disruption and ELM Erosion, Appendix E9, Section 1.7 (Divertor), ITER Design Description Document, ITER No. G 17 DDD 1 96-08-21 W2.1, August 1996.

[8] T. Ozeki, M.S. Chu, L.L. Lao et al., Nucl. Fusion 30 (1990) 1430.

[9] V.V. Parail, B. Balet, P. Bak et al., In: Proc. 22nd EPS Conf. on Controlled Fusion and Plasma Physics, Bournemouth, 1995, p. I-013.

[10] J. Lingertat, B. Alper, S. Ali-Arshad et al., in: Proc. 22nd EPS Conf. on Controlled Fusion and Plasma Physics, Bournemouth, 1995, p. III-281.

[11] M.A. Mahdavi, D.N. Hill, S.L. Allen et al., J. Nucl. Mater. 162-164 (1989) 245.

[12] T.H. Osborne, R.J. Groebner, L.L. Lao et al., Plasma Phys. Control. Fusion 40 (1998) 845.

[13] Yu. Igitkhanov, G. Janeschitz, G.W. Pacher, IAEA Technical Committee Meeting on H-mode Physics, Closter Seeon, Germany, 1997.

[14] T.H. Osborne, A.W. Leonard, private communications, 1998.

[15] A.W. Leonard, W. Suttrop, T.H. Osborne, J. Nucl. Mater. 241-243 (1997) 628.

[16] W. Suttrop, K. Schonmann, J. Schweinzer et al., in: Proc. 22nd EPS Conf. on Controlled Fusion and Plasma Physics, Bournemouth, 1995, p. III-237.

[17] R. Mohanti, C. Brickley, J.P. Christiansen et al., in: Proc. 23rd EPS Conf. on Controlled Fusion and Plasma Physics, Berchtesgaden 1995, p. I-101.

[18] S.J. Fielding, K.B. Axon, M.G. Booth et al., J. Nucl. Mater. 241-243 (1997) 902.

[19] Y. Kamada, K. Ushigusa, O. Naito et al., Plasma Phys. Control. Fusion 36 (1994) A123.

[20] V.P. Bhatnagar, D. Bartlett, S. Clement et al., in: Proc. 23rd EPS Conf. on Controlled Fusion and Plasma Physics, Berchtesgaden, 1997, p. I-77. 
[21] H. Zohm, Plasma Phys. Control. Fusion 38 (1996) 105.

[22] D.N. Hill, R. Ellis, W. Ferguson et al., Rev. Sci. Instr. 59 (1988) 1878.

[23] A. Herrmann, W. Junker, K. Gunther et al., Plasma Phys. Control. Fusion 37 (1995) 17.

[24] D.P. Coster, B. Braams, J. Neuhauser et al., in: Proc. 21st EPS Conf. on Controlled Fusion and Plasma Physics, Montpellier, 1994, p. II-846.

[25] P. Franzen, R. Behrisch, C. Garcia-Rosales et al., Nucl. Fusion 37 (1997) 1375.

[26] J.P. Coad, M. Rubel, C.H. Wum, J. Nucl. Mater. 241-243 (1997) 408.

[27] C.P.C. Wong, D.G. Whyte, R.J. Bastasz, J. Nucl. Mater. 258-263 (1998) 433.

[28] S. Ali-Arshad, A. Edwards, J. Lingertat, et al., in: Proc. 23rd EPS Conf. on Controlled Fusion and Plasma Physics, Kiev, 1996, p. I-56.

[29] J. Lingertat, private communications, 1998.

[30] J. Lingertat et al., ELM dynamics and scaling, in: The Fourth European Physics Worshop, Stockholm, 1996.

[31] A. Herrmann, P. Franzen, W. Herrmann et al., in: Proc. 23rd EPS Conf. on Controlled Fusion and Plasma Physics, Berchtesgaden, 1997, p. IV-1417.
[32] R. Schneider, D. Reiter, D. Coster et al., J. Nucl. Mater. 220-222 (1995) 1076.

[33] D.P. Coster, R. Schneider, J. Neuhauser et al., J. Nucl. Mater. 241-243 (1997) 690.

[34] J.P. Coad, Plasma viewing in JET using endoscopes and a detailed design for ITER, in: G. Gorini, E. Sidoni, P.E. Stott (Eds.), Diagnostics for Experimental Thermonuclear Fusion Reactors 2, Plenum, New York, 1998, Proc. International Workshop on Diagnostics for ITER, Varenna, Italy, September 1997.

[35] E. Gauthier, A. Chankin, S. Clement et al., in: Proc. 23rd EPS Conf. on Controlled Fusion and Plasma Physics, Berchtesgaden, 1997, p. I-61.

[36] J. Lingertat, A. Tabasso, S. Ali-Arshad et al., J. Nucl. Mater. 241-243 (1997) 402.

[37] The JET Team, Plasma Phys. Control. Fusion 39 (1997) B1.

[38] P. Kupschus, J.C.M. de Hass, M. Gadeberg et al., in: Proc. 23rd EPS Conf. on Controlled Fusion and Plasma Physics, Berchtesgaden, 1997, p. I-45. 